\title{
MICHEL FOUCAULT: SUJETO E HISTORIA
}

\section{Edgardo Castro*}

Como a propósito de numerosas problemáticas centrales del pensamiento del siglo $\mathrm{XX}$, también acerca de la historia nos encontramos con expresiones provocativas, $\mathrm{y}$ a veces hasta contradictorias, por parte de M. Foucault: "Yo no soy verdaderamente un historiador. $Y$ no soy un novelista. Practico una especie de ficción histórica"1, "mis libros no son tratados de filosofía ni estudios históricos; en el mejor de los casos, ellos son fragmentos filosóficos en los canteros de la historia"2. Más allá de estas declaraciones u otras del mismo género, es innegable que la historia, como objeto y como práctica, ha sido una de las preocupaciones y uno de los ejes mayores del trabajo de Foucault. Para comprenderlo, basta con darse cuenta de la estrecha relación que vincula, en su pensamiento, la problemática de la historia con lo que él considera el verdadero tema de sus investigaciones: el sujeto $^{3}$. Así, lo que denomina arqueología (como descripción de las condiciones históricas de posibilidad del saber) y el concepto de episteme serán sus recursos metodológicos a la hora de mostrar cómo el hombre se ha constituido, al mismo tiempo, en sujeto y objeto de conocimiento. La noción de dispositivo, por su parte, y la historia entendida como genealogía serán la estrategia para describir las relaciones de poder, es decir, las relaciones entre sujetos. Finalmente, para estudiar las relaciones ya no entre sujetos, sino del sujeto consigo mismo, las prácticas de subjetividad, Foucault concebirá su trabajo como una historia de las prácticas.

En el presente trabajo, nos proponemos recorrer las etapas centrales de la problemática de la historia-sujeto en los trabajos de M. Foucault y mostrar, a través de ellas, cómo nuestro autor ha renovado, sobre todo, la práctica filosófica de la historia y el modo de concebirla.

Profesor de las Universidades Nacionales de La Plata, San Martín y de la Universidad Nacional de Rosario. edgardocastro@fibertel.com.ar

1 Foucault, Michel, Dits et écrits, Gallimard, Paris, 1994, vol. IV, p. 40. Las traducciones son nuestras.

2 Ibid., vol. IV, p. 21.

${ }^{3}$ Ibid., p. 223. 


\section{Historia y locura}

Desde 1954 (Maladie mentale et personnalitê) hasta 1961 (Histoire de la folie à l'âge classique), como lo sugieren los títulos de sus libros, el trabajo de Foucault tuvo como campo de referencia los saberes acerca de la enfermedad mental y la locura. Es en este ámbito donde aparece la relación entre historia y sujeto.

El capítulo tercero de Maladie mentale et personnalité se titula, en efecto, "La enfermedad mental y la historia individual". Aquí, sirviéndose en gran medida de una psicología de corte marxista, Foucault busca explicar cómo la enfermedad mental se origina en las contradicciones concreta del individuo. Para ello y para articular ambas problemáticas, la del sujeto, en este caso enfermo, y la de la historia, Foucault se sirve del concepto de alienación. Así, la alienación histórica aparece como la condición de la alienación psicológica. Para Foucault, la psicología de Pavlov permite pensar el paso de una a otra. Concluye Foucault: 'No es, entonces, porque se está enfermo que se es alienado, sino en la medida en que se es alienado, se está enfermo"4.

Desde este punto de vista, Foucault critica al psicoanálisis en la medida en que no ha distinguido suficientemente, en relación la enfermedad mental, entre evolución (el desarrollo de la naturaleza) e historia (los conflictos socio-culturales) $)^{5}$.

En Histoire de la folie (1961) y en Maladie mentale et psychologie (1962), reedición de Maladie mentale et personnalité a luz de los resultados de la Histoire de la folie, la relación entre historia y sujeto será pensada en otros términos. "Maladie mentale et personnalité es una obra completamente separada de todo cuanto escribí posteriormente. La escribí en un período en el que las diferentes significaciones del término 'alienación', su sentido sociológico, histórico y psiquiátrico, se confundían en una perspectiva fenomenológica, marxista y psiquiátrica. Actualmente no hay ningún nexo entre estas nociones $\left[[. . .]^{p 6}\right.$.

\footnotetext{
${ }^{4}$ Foucault, Michel, Maladie mentale et personnalité, PUF, Paris, 1954, p. 103.

${ }^{5}$ Ibid., p. 37.

${ }^{6}$ Foucault, Michel, Dits et écrits, Gallimard, Paris, 1994, vol. IV, p. 665.
} 
En Histoire de la folie, Foucault distingue cuatro formas de conciencia de la locura: la conciencia crítica (la oposición inmediata, sin conceptos ni definiciones, a la locura), la conciencia práctica (la oposición a la locura a partir de un grupo que se considera portador de las normas de la razón), la conciencia enunciativa (la aprehensión perceptiva de la locura) y la conciencia analítica (la conciencia de la locura a nivel del saber). Cada figura histórica de la locura, cada experiencia de la locura, la del Renacimiento, la de la Época clásica (siglos XVII y XVIII) y la de la Modernidad implica a la vez la unidad y el conflicto entre estas cuatro formas de conciencia de la locura. Una experiencia de la locura será, entonces, necesariamente la historia de esas experiencias del límite por las que una cultura rechaza lo que será para ella lo Exterior, lo Otro. Ya no se trata de la historia dialéctica de la alienación, sino de una historia de experiencias de la locura que ponen de relieve las estructuras de lo trágico, de las separaciones irreconciliables, de los enfrentamientos que perduran. La Histoire de la folie es, en este sentido, una historia de las experiencias de la locura en términos trágicos ${ }^{7}$.

Respecto de la relación entre sujeto e historia, Histoire de la folie marca un cambio fundamental en el trabajo de Foucault. Por un lado, no sólo las formas de la locura y de la enfermedad mental son abordadas a partir de las condiciones históricas, también las formas del saber sobre la locura. Por otro, la historicidad del saber sobre la locura no se explica en relación con la mayor o menor objetividad que éste pueda alcanzar, en una historia del conocimiento concebida en términos teleológicos (como manifestación progresiva de una verdad), sino por las diferentes configuraciones históricas, discontinuas, que ponen de relieve las diferentes experiencias de la locura. Estas configuraciones históricas, es importante subrayarlo, no son sólo el producto del saber; en ellas, las prácticas discursivas, como dirá más adelante, se entrelazan con las prácticas no-discursivas (las instituciones, los reglamentos, las formas sociales, etc.).

7 Posteriormente, Foucault será crítico respecto del concepto de experiencia utilizado en esta obra cf. Foucault Michel, Dits et écrits, Gallimard, Paris, 1994, vol. I, p. 159. 


\section{La madre de las ciencias humanas}

Los trabajos posteriores de Foucault (Naissance de la clinique, de 1963, Les Mots et les choses, de 1966 y L'Archéologie du savoir, de 1969) pueden ser vistos como una profundización de los resultados de la Histoire de la folie, como una ampliación de la problemática de la historicidad del saber. Pero ello no implica una mera continuidad. Por un lado, Foucault ya no sólo se ocupará de los saberes sobre lo Otro, sobre la locura, sino sobre lo Mismo, sobre la razón. Por otro lado, el estudio de las condiciones históricas del saber no seguirá inmediatamente la parte final de la Histoire de la folie, es decir, el abordaje del análisis histórico de los nexos entre prácticas discursivas y no discursivas en la época de la razón de estado y del liberalismo. Pero Foucault volverá más tarde sobre esta cuestión y esta época, cuando aborde el estudio de la racionalidad política moderna.

Presentando las cosas esquemáticamente, se puede decir que en la Histoire de la folie, bajo la influencia de Nietzsche entre otros, Foucault se distancia de una de la corrientes que más influyeron en sus años de formación, el marxismo, y de su concepción dialéctica de la historia. Ahora, abordando la historicidad de la razón y, más precisamente, de la racionalidad moderna, Foucault se distanciará de la fenomenología, la otra corriente determinante en su formación. Más allá de las polémicas a que ha dado lugar la relación de Foucault con el estructuralismo y de sus declaraciones al respecto, resulta innegable que el estructuralismo ha marcado en gran medida su alejamiento de la fenomenología y también, de alguna manera, su concepción de lo que denominó arqueología.

Se pude afirmar, con todas las precauciones de las esquematizaciones, que la fenomenología describe la historia del conocimiento con una visión horizontal del desarrollo de la racionalidad. En esta perspectiva, a partir del momento en que se constituye una determinada idealidad (las nociones de la geometría, por ejemplo), en y por la subjetividad, se inicia una tradición de sentido que tiende teleológicamente a desplegar toda su verdad. Foucault, en cambio, no busca exponer cómo la historia de una determinada disciplina resulta inteligible a partir de la tradición de sentido que la anima desde sus actos fundadores, sino, al contrario, en mostrar cómo sus condiciones de surgimiento o, para utilizar su vocabulario, su emergencia responde, más bien, a condiciones históricas de posibilidad que, por un lado, son comunes a todos o a varios saberes de una época $y$, por otro, que cambian de una 
época a otra. Con la noción de episteme, el saber no es analizado desde la perspectiva de su forma racional o de su objetividad, sino desde el punto de vista de la homogeneidad discontinua que rige la formación de diferentes discursos pertenecientes a una misma época.

En Les Mots et les choses, Foucault describe la configuración de las epistemes renacentista, clásica y moderna. Mientras la primera, la episteme renacentista, estaba dominada por la semejanza (que regía tanto al lenguaje como a las cosas), a comienzos del siglo XVII, ella se convierte en la ocasión y en el lugar del error ${ }^{8}$. Por ello, su lugar será ocupado, en la episteme clásica, por el orden. La tarea del pensamiento consistirá, entonces, en elaborar un método de análisis universal (mátbesis y taxonomía) que, estableciendo un orden cierto entre las representaciones y los signos, refleje el orden del mundo.

Ahora bien, Les Mots et les choses lleva como subtítulo Una arqueología de las ciencias bumanas. Todo el recorrido de Foucault en esta obra está dirigido a mostrar cómo fueron posibles las ciencias humanas (la psicología, la sociología, el análisis literario), cuáles fueron sus condiciones históricas de posibilidad en la episteme moderna.

Según Foucault, nos encontramos en la modernidad con un triedro de saberes conformado por las llamadas ciencias formales (como la matemática y la lógica), las ciencias empiricas (la economía política, la biología y la filología) y la analitica de la finitud. Las ciencias humanas no se ubican en ninguno de los tres dominios definidos por estas ciencias, sino en el espacio de encuentro entre ellos. Aunque ellas se sirvan a veces de procedimientos de formalización, emparentados con las ciencias precisamente formales, lo que las define propiamente es la relación que ellas mantienen con las ciencias empíricas y la analítica de la finitud: se ubican en el espacio que va de las ciencias empíricas a la analítica de la finitud. Su espacio está delimitado por lo que en el hombre hay de positivo (el trabajo, la vida y el lenguaje) y lo que posibilita al hombre saber qué es el trabajo, la vida y el lenguaje. Expresándolo con otros términos, las ciencias humanas han sido posibles a partir del momento en que el hombre se ha convertido en objeto finito y en sujeto finito del saber.

${ }^{8}$ Cfr.: Foucault , Michel, Les Mots et les choses, Gallimard, Paris, 1966, pp. 65-68. 
Con analítica de la finitud, nuestro autor se refiere precisamente al esfuerzo por pensar lo finito a partir de lo finito; y ello define la disposición fundamental de la filosofía moderna. Foucault distingue tres grandes figuras de la analítica de la finitud: la oscilación entre lo empirico y lo trascendental (M. Merleau-Ponty), la oscilación entre el cogito y lo impensado (Husserl) y la oscilación entre el retroceso y el retorno del origen (Heidegger).

Por extraño que pueda parecer -y el mismo Foucault lo hace notar-, la historia no figura entre de las ciencias humanas; es presentada, sin embargo, como la primera y la madre de todas las ciencias del hombre?. Lo que el Orden ha sido para la episteme clásica -la forma y el contenido de lo que puede ser conocido con certeza-, la Historia lo ha sido para la episteme moderna. La historia, el flujo de la temporalidad, definirá tanto el modo de ser de las empiricidades como aquello a partir de lo cual éstas son posibles, el modo de ser de todo lo que nos es dado en la experiencia. 'La filosofía del siglo XIX se alojará en la distancia de la historia a la Historia, de los acontecimientos al Origen, de la evolución al primer brote de la fuente, del olvido al Retorno. Ya no será entonces Metafísica sino en la medida en que sea Memoria, y necesariamente reconducirá el pensamiento a la cuestión de saber lo que es para el pensamiento tener una historia. Esta cuestión urgirá sin cesar la filosofía de Hegel a Nietzsche y más allá. No veamos aquí el fin de una reflexión filosófica autónoma, demasiado matinal y demasiado orgullosa para inclinarse, exclusivamente, sobre lo que ha sido dicho antes de ella y por otros; no hagamos de ello un pretexto para denunciar un pensamiento impotente para mantenerse de pie solo y siempre obligado a enrolarse en un pensamiento ya concluido. Basta con reconocer aquí una filosofía desprendida de cierta metafísica en tanto se ha despegado del espacio del orden, pero destinada al Tiempo, a su flujo, a sus retornos, porque está capturada en el modo de ser de la Historia"10.

Como las ciencias humanas, también la historia oscila entre los límites establecidos por la analítica de la finitud: por un lado, nos encontramos con una historicidad de las empiricidades y, por otro, con una historicidad trascendental a partir de la cual es posible la historicidad de las empiricidades. Desde el punto de vista de las empiricidades, nos encontramos con una fragmentación de la historia, con un hombre

\footnotetext{
${ }^{9}$ Ibid, p. 378.

${ }^{10}$ Ibid., pp. 231-232.
} 
deshistorizado ${ }^{11}$. La época clásica había pensado la historia como unitaria, ya fuera porque proyectaba la temporalidad de la naturaleza sobre lo humano $o$, inversamente, porque subsumía toda temporalidad en la historicidad humana concebida según un modelo providencial. Con los saberes modernos la historia se fragmenta, aparece una temporalidad propia de la vida, otra del trabajo, otra del lenguaje. Desde un punto de vista trascendental, en cambio, esta historicidad desnuda del hombre aparece como su historicidad propia y como fuente de la dispersión radical de todas las formas históricas. Aparece también la necesidad de buscar y pensar las leyes de esta forma pura de la temporalidad.

Les mots et les choses es, por ello, una arqueología de las ciencias humanas y también de la madre de las ciencias humanas, de la historia.

Como sabemos, Les Mots et les choses concluye con la proclamación de la muerte del hombre, con la desaparición del sujeto moderno, tal como lo han concebido la filosofía (particularmente las diferentes formas de la fenomenología) y las ciencias humanas. Foucault sostiene que la figura del hombre se formó a partir de la desaparición del discurso clásico, en los intersticios de un lenguaje en fragmentos. La reaparición del ser del lenguaje en la literatura, en la lingüística, en el psicoanálisis, en la etnología, nos anuncia que la figura del hombre está por descomponerse.

Foucault no proclama la muerte de la historia, pero, al menos la historia del conocimiento, en la medida en el que el sujeto ha muerto, debe convertirse en arqueología. El sujeto, la conciencia, en efecto, ya no podrá ser el fundamento que garantice la continuidad en el tiempo de la racionalidad y de la verdad. La historia ya no podrá ser pensada ni en términos hegelianos ni husserlianos. Ella deberá ser abordada en su dispersión.

\section{De la episteme al dispositivo y a las prácticas}

Como lo reconocerá el propio Foucault posteriormente, la noción de episteme de Les mots et les choses plantea no pocas dificultades. Por ello, tres años más tarde, en L'Archéologie du savoir, y para deshacerse de todo resabio estructuralista, buscará definir el nivel de la descripción arqueológica, de la episteme, a partir del concepto de formación discursiva.

11 Ibid., pp. 380. 
Una formación discursiva es "un conjunto de reglas anónimas, históricas, siempre determinadas en el tiempo y en el espacio, que han definido en una época dada, y para un área social, económica, geográfica o lingǘstica dada, las condiciones de ejercicio de la función enunciativa" 12 . Describir una formación discursiva, entonces, será afrontar un dominio de análisis constituido por todos los enunciados efectivamente dichos o escritos, en su dispersión de acontecimientos y en su singularidad.

Para definir el nivel propio de la descripción arqueológica de los enunciados, Foucault lo distingue del análisis lingüístico y del análisis de la historia del pensamiento. También los lingüistas trabajan con enunciados y los describen, pero en este caso se trata de establecer aquellas reglas que permitirían eventualmente construir nuevos enunciados. Para la arqueología el problema es otro: no según cuáles reglas es posible construir nuevos enunciados, sino cómo ha sucedido que sólo tales enunciados hayan existido y no otros. La historia del pensamiento, por su parte, busca encontrar, más allá de los enunciados o a partir de ellos, la intención del sujeto hablante, sus actividades conscientes $\mathrm{o}$ inconscientes. $\mathrm{El}$ análisis arqueológico de los enunciados no los remite a una instancia fundadora, sino sólo a otros enunciados, para mostrar sus correlaciones, sus exclusiones, etc. Se podría decir, entonces, que el enunciado es una proposición o una frase considerada desde el punto de vista de sus condiciones de existencia, no como proposición o como frase.

Pero esta elaboración metodológica del concepto de episteme no será suficiente para nuestro autor. En efecto, la arqueología permite describir los discursos de las diferentes epistemes, pero, encerrada en el orden de lo discursivo, no podía describir los cambios en sí mismos, sino sólo en sus resultados. Como sostiene el propio Foucault, le faltaba a su trabajo el análisis del poder, de la relación entre lo discursivo y lo no-discursivo. A esta necesidad responde la introducción del concepto de dispositivo. El dispositivo es, en definitiva, más general que la episteme, que podría ser definida como un dispositivo exclusivamente discursivo. La descripción de los dispositivos es uno sentidos posibles de la genealogía foucaultiana.

Aunque no encontramos ninguna definición al respecto, podemos delimitar la noción de dispositivo como sigue: 1) El dispositivo es la red de

${ }^{12}$ Foucault, Michel, L'Archéologie du savoir, Gallimard, Paris, 1969, pp. 153-154. 
relaciones que se pueden establecer entre elementos heterogéneos: discursos, instituciones, arquitectura, reglamentos, leyes, medidas administrativas, enunciados científicos, proposiciones filosóficas, morales, filantrópicas, lo dicho y lo no-dicho. 2) El dispositivo establece la naturaleza del nexo que puede existir entre estos elementos heterogéneos. Por ejemplo, el discurso puede aparecer como programa de una institución, como un elemento que puede justificar u ocultar una práctica, o funcionar como una interpretación a posteriori de esta práctica, ofrecerle un campo nuevo de racionalidad. 3) Se trata de una formación que en un momento dado ha tenido por función responder a una urgencia. El dispositivo tiene así una función estratégica, como, por ejemplo, la reabsorción de una masa de población flotante que era excesiva para una economía mercantilista. 4) Además de definirse por la estructura de elementos heterogéneos, un dispositivo se define por su génesis. Foucault distingue al respecto dos momentos esenciales: un primer momento del predominio del objetivo estratégico; un segundo momento de la constitución del dispositivo propiamente dicho. 5) El dispositivo, una vez constituido, permanece tal en la medida en que tiene lugar un proceso de sobredeterminación funcional: cada efecto, positivo o negativo, querido o no-querido, entra en resonancia o contradicción con los otros y exige un reajuste.

En lo que algunos denominan el "último Foucault", el de los trabajos sobre la ética, nuestro autor aborda los modos en que el sujeto se instaura a sí mismo como sujeto. Aquí la noción de dispositivo es englobada en una más amplia: práctica. Por ello, su ética puede ser caracterizada como una historia de las prácticas de subjetivación.

A pesar de la importancia que este concepto tiene en las obras de Foucault, tampoco encontramos en éstas ninguna exposición detallada del concepto de práctica; resulta necesario reconstruirlo a partir de otras indicaciones. El primer texto a tomar en consideración para tal fin es "Qu'est-ce que les Lumières?"13. En este texto Foucault plantea la necesidad de considerar la modernidad como un éthos, es decir, como una actitud. Esta actitud debe traducirse, según Foucault, en una serie de investigaciones a la vez arqueológicas y genealógicas acerca de las prácticas que nos constituyen históricamente como sujetos. Foucault atribuye a estas investigaciones tres

${ }^{13}$ Foucault, Michel, Dits et écrits, Gallimard, Paris, 1994, vol. IV, pp. 562-578. 
características que, en definitiva, delimitan y definen lo que entiende por práctica. 1) Homogeneidad. Estas investigaciones no se ocupan de las representaciones que los hombres tienen de sí mismos o de las condiciones que los determinan, sino más bien de "lo que hacen y la manera en que lo hacen"; más precisamente aún, de "las formas de racionalidad que organizan las maneras de hacer"14. A propósito de la racionalidad o regularidad de las prácticas, Foucault habla de "aspecto tecnológico". También es necesario analizar la libertad con la que los sujetos actúan en este sistema de prácticas: aquello que Foucault denomina el "juego estratégico". 2) Sistematicidad. Estas investigaciones exigen además que se considere sistemáticamente el dominio de las prácticas, es decir, que se tome en consideración el eje del saber (las prácticas discursivas), del poder (las relaciones con los otros) y de la ética (las relaciones del sujeto consigo mismo) en lo que tienen de específico y en su entrelazamiento. 3) Generalidad. Por último, las prácticas que Foucault estudia (las relaciones entre razón y locura, enfermedad y salud, crimen y ley) poseen un carácter recurrente. Sin embargo, no hay que interpretar esta generalidad o recurrencia como si se tratara de una realidad metahistórica cuya permanencia y variaciones habría que reconstruir a través de la historia. La generalidad de las prácticas es en sí misma una configuración histórica singular. En este sentido, Foucault habla de una reducción nominalista de la antropología filosófica.

Un segundo texto relevante es la introducción a L'Usage des plaisirs. La historia de la sexualidad no es una historia ni de los comportamientos ni de las representaciones, sino de una experiencia, "si se entiende por 'experiencia' la correlación entre dominios de saber, tipos de normatividad y formas de subjetividad en una cultura"15.

El tercer texto a considerar es la introducción general a la Histoire de la sexualité, "Préface à l'Histoire de la sexualité"16. Aquí Foucault interpreta el análisis histórico de las formas de experiencia en términos de "pensamiento". 'Por 'pensamiento' entiendo lo que instaura, en diferentes formas posibles, el juego de lo verdadero y de lo falso y, en consecuencia, constituye al ser humano como sujeto de conocimiento; lo que funda la aceptación o el rechazo de la regla y constituye al ser humano como sujeto

${ }^{14}$ Ibid., p. 576.

${ }^{15}$ Foucault, Michel, L'Usage des plaisirs, Gallimard, Paris, 1984, p. 10.

${ }^{16}$ Foucault, Michel, Dits et écrits, Gallimard, Paris, 1994, vol. IV, pp. 578-584. 
social o jurídico; lo que instaura la relación consigo mismo y con los otros y constituye al ser humano como sujeto ético. Así entendido, no hay que buscar el pensamiento en las formulaciones teóricas, como las de la filosofía o de la ciencia; éste puede y debe ser analizado en todas las maneras de decir, de hacer, de conducirse, en las que el individuo se manifiesta y obra como sujeto de conocimiento, como sujeto ético o jurídico, como sujeto consciente de sí y de los otros. En este sentido, el pensamiento es considerado como la forma misma de la acción, como la acción en la medida en que ésta implica el juego de lo verdadero y de lo falso, la aceptación o el rechazo de la regla, la relación consigo mismo y con los otros"17.

En síntesis, podemos decir que Foucault entiende por prácticas la racionalidad o la regularidad que organiza lo que los hombres hacen ("sistemas de acción en la medida en que están habitados por el pensamiento") ${ }^{18}$, que tiene un carácter sistemático (saber, poder, ética) y general (recurrente), y que por ello constituye una "experiencia" o un "pensamiento".

\section{Biohistoria}

Contemporáneamente a su descripción histórica de las prácticas de subjetividad, Foucault se ocupó de la formación de la racionalidad política moderna. En Vigilar y castigar (1975), Foucault se había ocupado de la formación y del funcionamiento del dispositivo disciplinario: una forma de ejercicio del poder que tiene por objeto los cuerpos individuales y que busca hacerlos políticamente dóciles y económicamente provechosos. En la última parte de La voluntad de saber (1976), luego de haber analizado el dispositivo de sexualidad, Foucault describe otra forma de ejercicio del poder que también tiene por objeto el cuerpo, pero no el individual, sino el de la especie, el de la población, el cuerpo colectivo. Se trata de la biopolítica. La publicación de los cursos en el Collège de France, algunos de ellos recientemente (Sécurité, territoire population y Naissance de la bioipolitique), a puesto de relieve la importancia de la biopolítica en lo que se podría denominar su filosofía política.

${ }^{17}$ Ibid., pp. $579-580$.

${ }^{18}$ Ibid., p. 580. 
La formación de una biopolítica, de una política de la vida biológica, marca, según Foucault, el umbral de la modernidad biológica. Con sus palabras, si, para Aristóteles, el hombre era un animal viviente y además capaz de una existencia política; el hombre moderno es el animal cuya política tiene por objeto su ser viviente.

Según Foucault, esta posibilidad de intervención del hombre sobre la vida abre el capítulo de una biohistoria. "En nuestros días se descubre un hecho nuevo: la historia del hombre y la vida están profundamente entrelazadas. La historia del hombre no continúa simplemente la vida, no se contenta simplemente con reproducirla, sino que la retoma, hasta cierto punto, y puede ejercer sobre su proceso cierto número de efectos fundamentales"19.

Para concluir, se puede resumir el sentido general del trabajo de Foucault en los canteros de la historia, retomando sus propias palabras: 'Todo lo que nos es propuesto, en nuestro saber, como de validez universal respecto de la naturaleza humana o de las categorías que es posible aplicar al sujeto exige ser verificado y analizado [...]. La primera regla del método para este género de trabajo es pues ésta: contornear lo más que se pueda, para interrogarlos en su constitución histórica, los universales antropológicos $(\mathrm{y}$, por supuesto, también los de un humanismo que haría valer los derechos, los privilegios y la naturaleza de un ser humano como verdad inmediata e intemporal del sujeto)"20.

\section{Resumen}

Este artículo describe la evolución del tema del sujeto en la obra de Michel Foucault. Más precisamente, desarrolla la relación entre sujeto e historia desde sus primeros trabajos, dedicados a la historia de la psicología y de la locura, hasta sus cursos consagrados a la biopolítica.

Palabras clave: "Foucault", "sujeto", "historia", 'Tocura”, "ética”, "biopolítica”.

\footnotetext{
${ }^{19}$ Ibid., p. 48.

${ }^{20}$ Ibid., pp. 633-634.
} 


\begin{abstract}
This article describes the evolution of the theme of the subject in the work of Michel Foucault. More precisely, it develops the relationship between subject and history, from his first works, dedicated to the history of psychology and of madness, to his courses devoted to biopolitics.
\end{abstract}

Key words: "Foucault", "subject”, "history", "madness", “ethics", "biopolitics". 